\title{
Fuel for Fun: a cluster-randomized controlled study of cooking skills, eating behaviors, and physical activity of 4 th graders and their families
}

Leslie Cunningham-Sabo ${ }^{1 *}$, Barbara Lohse ${ }^{2}$, Stephanie Smith ${ }^{3}$, Ray Browning ${ }^{4}$, Erin Strutz ${ }^{5}$, Claudio Nigg ${ }^{6}$, Meena Balgopal ${ }^{7}$, Kathleen Kelly ${ }^{8}$ and Elizabeth Ruder ${ }^{2}$

\begin{abstract}
Background: Childhood obesity remains a serious concern in the United States and in many other countries. Direct experience preparing and tasting healthful foods and increasing activity during the school day are promising prevention approaches. Engaging parents and families remains an important challenge. Fuel for Fun: Cooking with Kids Plus Parents and Play is a multi-component school- and family-based intervention for 4th graders and their families intended to promote positive food and activity environments, policies and behaviors at the individual, family and school levels. This paper describes the design and evaluation plan.

Methods/Design: Four cohorts of 4th-graders and their parents from 8 schools in 2 districts in the same Northern Colorado region are participating in a 4-arm cluster randomized controlled trial. Theory-based Fuel for Fun consists of 5 components delivered over 1 school year: 1) Cooking with Kids - Colorado; an experiential classroom-based cooking and tasting curriculum, 2) Cafeteria Connections; cafeteria-based reinforcements of classroom food experiences using behavioral economic strategies, 3) SPARK active recess; a playground intervention to engage children in moderate to vigorous activity, 4) Fuel for Fun Family; multi-element supports targeting parents to reinforce the 3 school-based components at home, and 5) About Eating; an online interactive program for parents addressing constructs of eating competence and food resource management. Outcomes include child and parent measures of fruit and vegetable preferences and intake, cooking, physical activity, sedentary behaviors and attitudes. School level data assess lunch plate waste and physical activity at recess. In-depth diet and accelerometry assessments are collected with a subsample of parent-child dyads. Data are collected at baseline, immediately post-intervention at 7 months, and at 12 month follow-up. We anticipate recruiting 1320-1584 children and their parents over the length of the project.

Discussion: The Fuel for Fun study design allows for impact assessment of school-, family- and online parent-based intervention components separately and in combination. Study strengths include use of theory- and evidence-based programs, valid child and parent self-report instruments, and objective measures of food, cooking, and physical activity behaviors at the individual, family and school levels. Parent involvement and engagement is examined through multiple strategies.
\end{abstract}

Trial registration: Clinicaltrials.gov registration number NCT02491294. Registered 7 July, 2015.

Keywords: Children, Cooking experience, Physical activity, Fruits and vegetables, Elementary schools, Parents, Experiential education, Eating competence, Online nutrition education, Family nutrition education

\footnotetext{
* Correspondence: Leslie.Cunningham-Sabo@Colostate.Edu

${ }^{1}$ Department of Food Science and Human Nutrition, Colorado State

University, 234 Gifford Building, Fort Collins, Colorado 80523-1571, USA

Full list of author information is available at the end of the article
} 


\section{Background}

Childhood obesity continues to be a major public health concern in the U.S. and elsewhere. School-based interventions addressing diet and physical activity have demonstrated promise [1]. Specifically, programs that include children in cooking and food preparation and increase physical activity during the school day are recommended to counter childhood obesity [2-4].

A recent systematic review of child-centered cooking programs identified eight articles meeting the inclusion criteria [5], of which two were rated strong in quality $[6,7]$. Both of these highly-rated studies were evaluations of the Cooking with Kids (CWK) curriculum, a school-based food and nutrition education curriculum that engages elementary school children in hands-on learning with fresh affordable foods based on diverse cultural traditions (http:// cookingwithkids.org/) [8]. Implementation of the full curriculum includes $16 \mathrm{~h}$ of cooking and tasting lessons throughout the school year, but allows for modifications to accommodate fiscal, time, and resource limitations. Participation in $C W K$ among predominantly Hispanic, $4^{\text {th }}$ grade children demonstrated gains in cooking self-efficacy among children with no previous cooking experience, and demonstrated increased preference for fruits and vegetables, especially for students receiving the cooking and tasting curriculum (as opposed to the curriculum with only tasting lessons). Moreover, gains in self-efficacy were particularly pronounced in male students [7]. Self-efficacy in cooking and fruit and vegetable preferences are relevant measures given that self-efficacy related to diet and nutrition is strongly correlated with dietary behavior [9], and fruit and vegetable preferences are often associated with intake [10, 11]. Similar gains with $C W K$ engagement were documented in a predominately non-Hispanic white sample of $4^{\text {th }}$ graders [6]. In that investigation of a $C W K$ program of shorter duration (10 h of cooking and tasting lessons), significant improvements in vegetable preferences, and cooking and food preparation attitudes and self-efficacy were affirmed with $C W K$ participation. As with the previous $C W K$ investigation, students who reported no prior cooking experience demonstrated the greatest improvement in these measures, and the effect was greatest in boys. Together, these studies support participation in $C W K$, and affirm the positive influence of a curriculum designed to allow students of diverse cultural backgrounds direct experience to foods through cooking or tasting activities.

Physical activity (PA) is the other cornerstone to energy balance. The school environment plays an important role in children's PA accumulation, but despite opportunities for PA during recess breaks and physical education classes, the sedentary nature of many school environments poses a challenge to school-day PA accumulation [12]. Interventions and other studies have reported that increasing school day PA accumulation increases whole-day PA in a magnitude greater than expected from the changes in school-day PA alone [4]. For instance, children encouraged to be physically active during the school day (e.g., during recess and physical education) have been shown to be more active after school than children whose PA was restricted during school [13]. Sports Play and Active Recreation for Kids (SPARK) is one program shown to increase students' moderate to vigorous PA during the school day [14].

Although energy balance may be as simple as "calories in" versus "calories out" from a physiologic standpoint, translating this principle to achieve improvements in public health is more complex. Successful programs and interventions aimed at obesity prevention must also address the context in which an individual's nutrition and physical activity choices are made $[15,16]$. For a pediatric audience, this includes the individual child, parents and family, school (including cafeteria) and community $[17,18]$. This type of system-wide approach engages multiple sectors of society to improve health and reduce overweight and obesity [15]. This approach stems from the Social Ecological Model (SEM) [19], and is central to the action plan for the Dietary Guidelines for Americans 2015-2020 [20].

Fuel for Fun: Cooking with Kids Plus Parents and Play $(F F F)$ is one such multiple-component, school and family intervention with a social-ecological approach. FFF incorporates the tested, validated $C W K$ program into wider school environments to include the cafeteria and facilitate family involvement. FFF builds on prior research to engage multiple sectors of the community to reduce the risk of childhood obesity by promoting healthful food and activity environments, policies and behaviors through: 1) developing and testing the efficacy of a 4th grade comprehensive school- and family-based intervention, 2) applying this intervention to an afterschool setting, and, 3) state-wide program dissemination. Two of these aims, applying this intervention to an after-school setting, and to a state-wide program dissemination, will not be described here. The purpose of this paper is to describe the study protocol for the FFF school- and family-based intervention.

\section{Methods/Design}

Fuel for Fun builds on our prior research and tenets of the Satter Eating Competence Model, Social Cognitive Theory (SCT), Experiential Learning Theory (ELT), SEM, Behavioral Economics, and Social Marketing [19, 21-25]. Over 4 years, annual cohorts participate in a 4-arm cluster randomized controlled trial that includes post-intervention follow-up. As shown in Fig. 1, cohorts 1 and 4 represent non-treatment with treatment occurring in cohorts 2 and 3. 


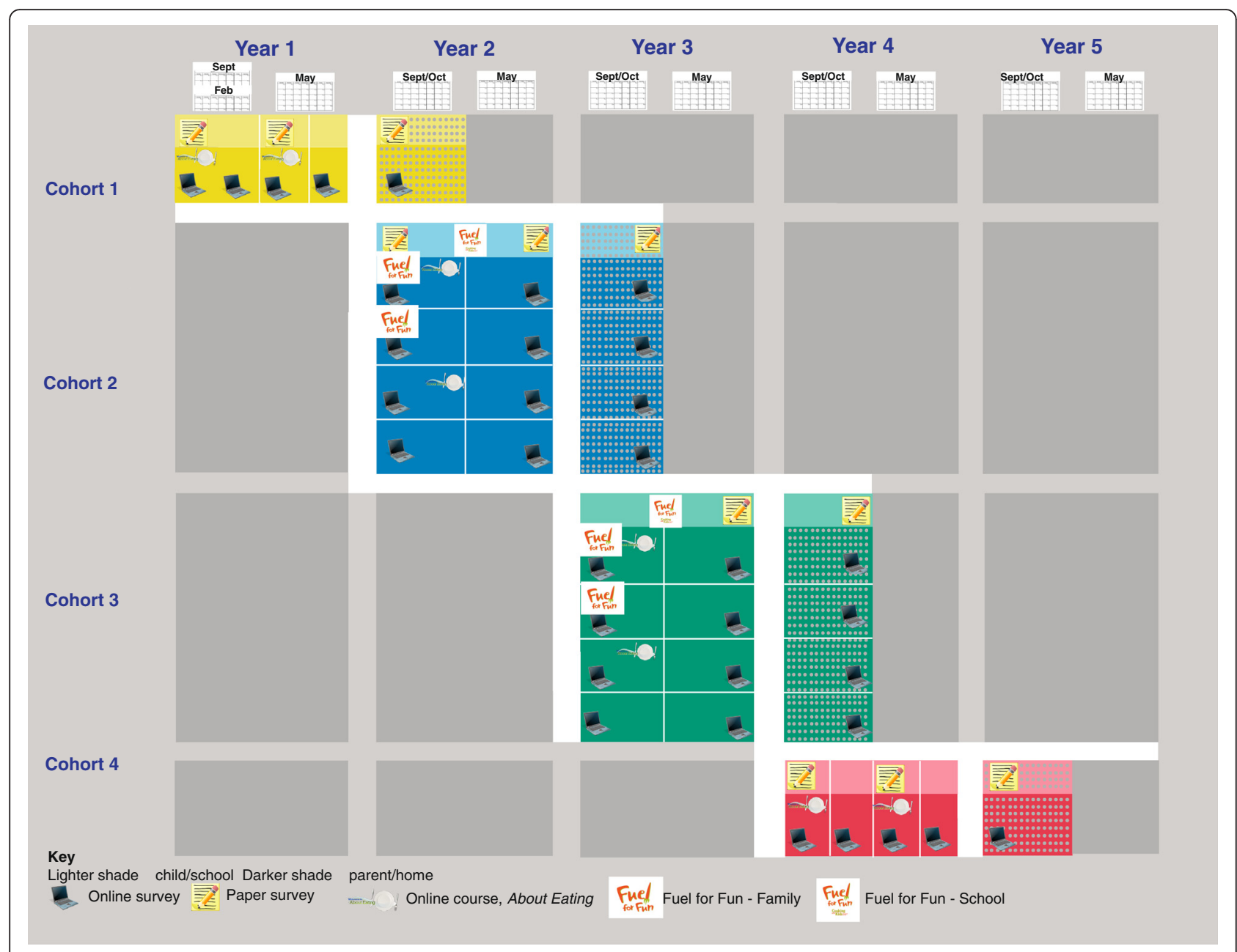

Fig. 1 Fuel for Fun: Cooking with Kids Plus Parents and Play Research Design. Fourth-grade students and their parents participate in this research study

Eight schools from 2 districts in the same Northern Colorado county were matched on percent of students receiving free/reduced price school meals, then randomly assigned to 1 of 4 arms so that 2 schools are in each of the following arms:

1) classroom, cafeteria, and playground components;

2) classroom, cafeteria, and playground components with multi-element family component;

3) classroom, cafeteria, and playground components with online parent education; and

4) classroom, cafeteria, and playground components with multi-element family component and online parent education.

\section{Setting and participants}

Approximately $554^{\text {th }}$ grade students are enrolled in each school annually. We anticipate 75-90 \% participation, thus totaling 330-396 students for each of the
4 years (1320-1584 over the length of the project). In one school district, $74 \%$ of students are white, $18 \%$ Hispanic, $3 \%$ Asian, and $1 \%$ African-American. In the second school district $75 \%$ of students are white, $20 \%$ Hispanic, $1 \%$ Asian, and $1 \%$ African American. Three of these 8 schools are assigned to participate in accelerometry assessment, and 100 parent-child dyads are recruited from all participating schools for indepth diet assessment. Consort charts for survey and diet assessment measures are shown in Figs. 2 and 3 for child and parent participation, respectively.

\section{Ethical approval and consent}

The study is approved by the Colorado State University, the Pennsylvania State University, and the Rochester Institute of Technology Institutional Review Boards and entities with similar functions within both school districts. All participating students and their parents sign assent/consent forms. 


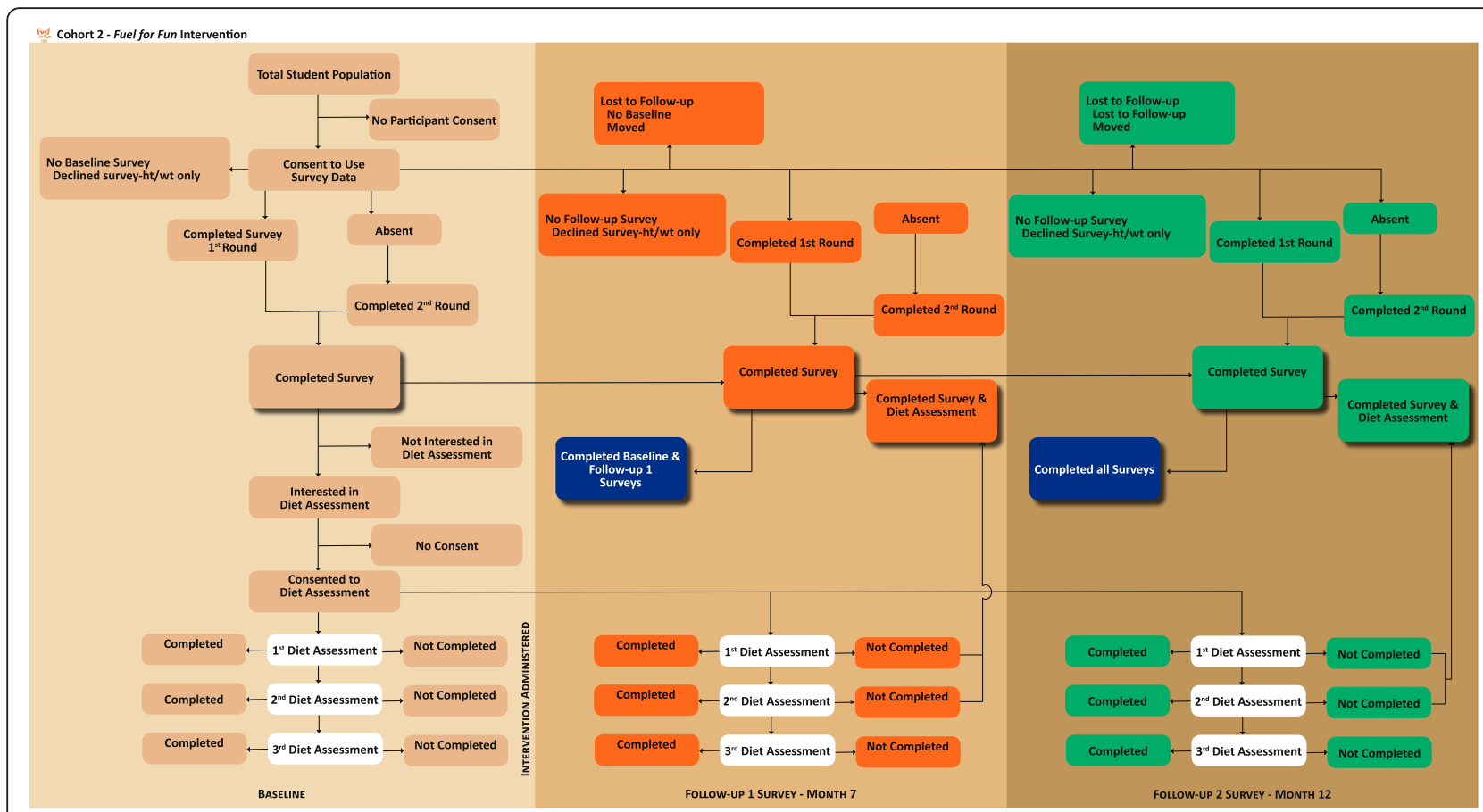

Fig. 2 Fuel for Fun child participation flowchart

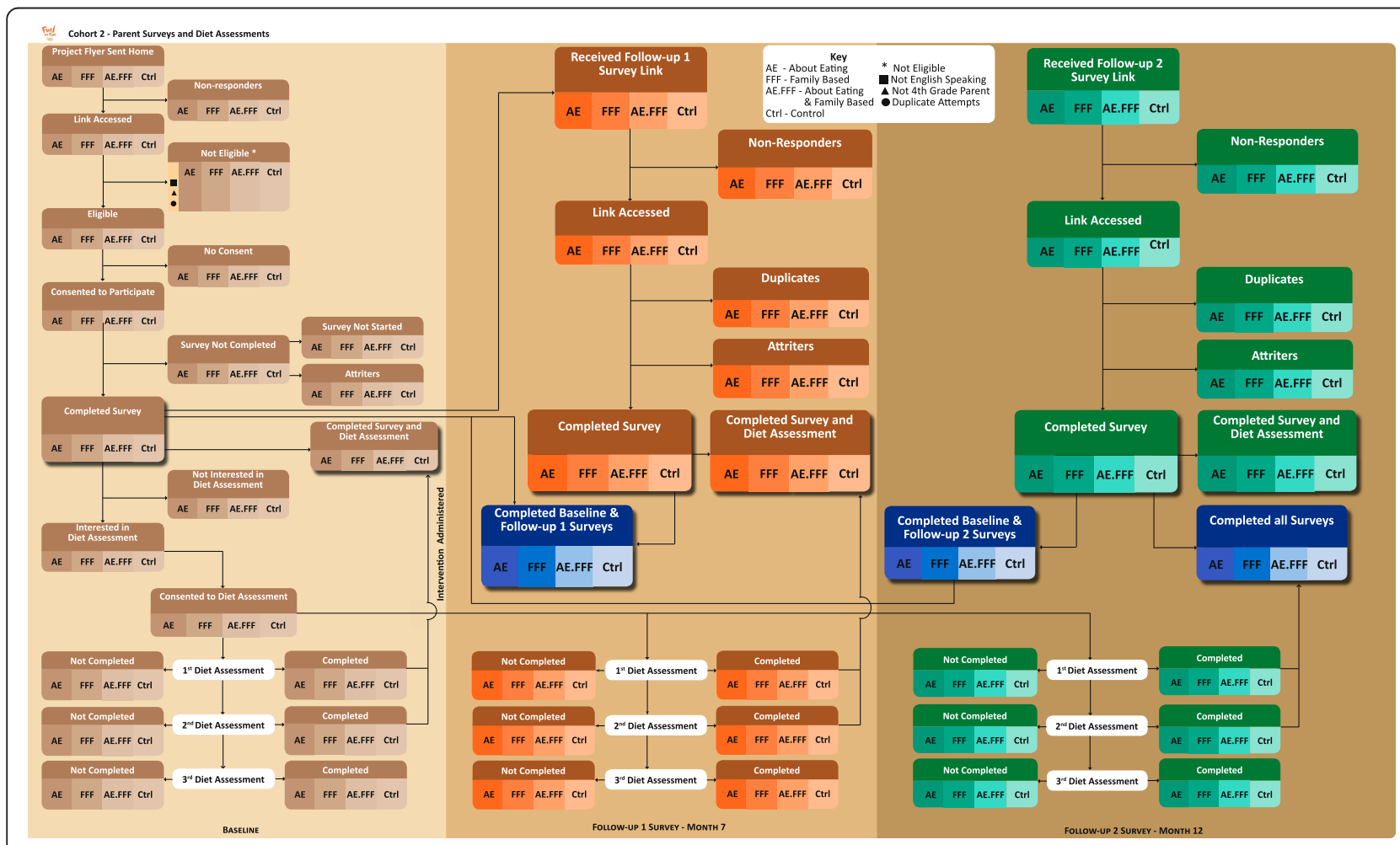

Fig. 3 Fuel for Fun parent participation flowchart 


\section{Intervention}

Co-investigators with branding expertise applied marketing strategies to generate a unique project name and logo. Name and logo selections were tested for understanding, clarity, and likeability by parents and children similar to the target audience. In testing, youth and adults described the logo as representing the scope of the project (i.e., "shows action and nutrition" in attention-demanding colors). After confirming no copyright infringement, we adopted Fuel for Fun: Cooking with Kids plus Parents and Play (Fig. 4) as the project name and logo.

Fuel for Fun consists of 5 components: 1) Cooking with Kids - Colorado; an experiential classroom-based cooking and tasting curriculum, 2) Cafeteria Connections; cafeteriabased reinforcements of classroom food experiences using behavioral economic strategies, 3) SPARK active recess; a playground intervention to engage all children in moderate to vigorous activity [26], 4) Fuel for Fun Family; multielement supports to reinforce the 3 school-based components at home targeting parents, and 5) About Eating; an online interactive program for parents addressing constructs of eating competence and food resource management [21]. Development and mode of delivery of each component is described below. The project logic model is depicted in Fig. 5.

\section{Intervention development}

The development of Fuel for Fun uses 3 strategies: 1) Design and development of new components; 2)

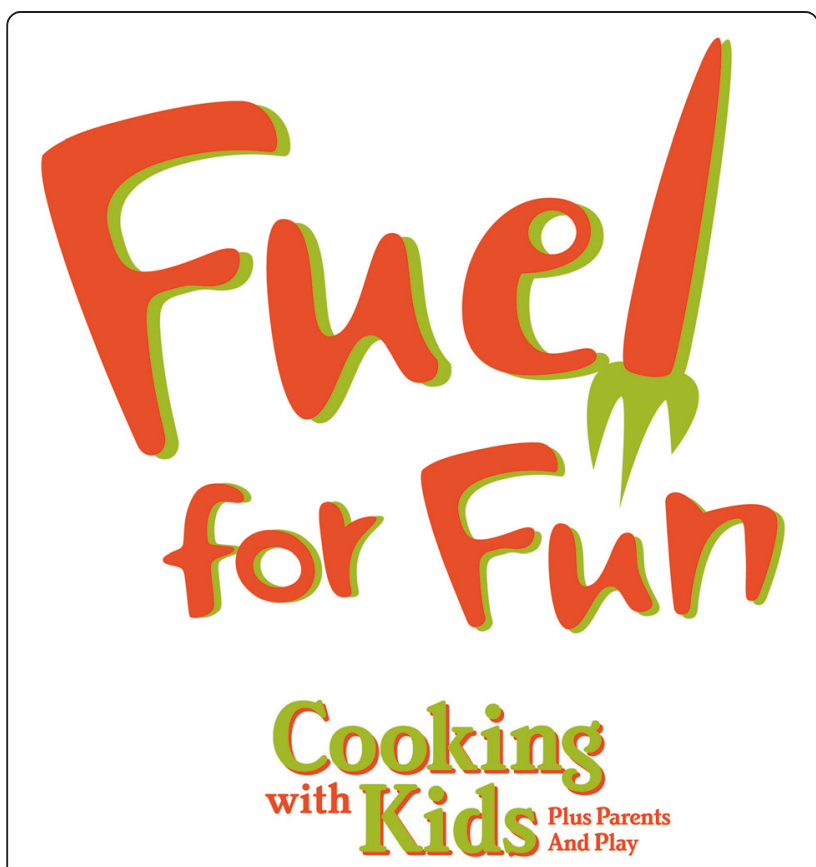

Fig. 4 Fuel for Fun: Cooking with Kids plus Parents and Play Logo adaptation of existing, tested activities and curricula; 3) integration of existing, stand-alone programs.

The study is adapted from and expands upon prior research examining the efficacy of the Cooking with Kids, Inc. program $(C W K)[6,7,27]$. For the experiential nutrition classroom component, the original CWK cooking and tasting lessons were reviewed and revised to more closely align with the state of Colorado academic standards and nutrition and education best practices. This 8month process involved a K-12 interdisciplinary curriculum expert, 5 educators and curriculum specialists from the participating school districts, the $C W K$ curriculum developers, and project nutrition education experts. Substantive changes included revising the lesson structure to be consistent with the Understanding by Design format [28] so that assessment strategies consistently address learning objectives and standards; greater emphases on indepth nutrition and mathematics knowledge and skills; regionally-relevant content (e.g., letters from local farmers); and more opportunities for differentiated instruction for diverse learning needs [29].

Integrating experiential classroom nutrition activities with cafeteria meals can increase fruit and vegetable consumption [30-32]. The FFF cafeteria component Cafeteria Connections - uses behavioral economic strategies [24, 33] to connect what children learn in the FFF classroom cooking and tasting lessons to foods served as part of the National School Lunch Program.

A companion family component was developed to reinforce the school-based intervention. It incorporated successful strategies identified through an extensive literature review, and included: family nights at school, home-based action packs (i.e., activity packets) for parent and child to complete together, and a parent blog. Subsequent telephone interviews with a representative sample of parents $(n=24 ; 41.7 \pm 5.7 y, 92 \%$ female, $91 \%$ White, $38 \%$ used $\geq 1$ food assistance program) of nonintervention $4^{\text {th }}$ graders explored these elements in greater detail. Parents preferred family events at school to be held toward the end of the work week (Thursdays or Fridays) and in the early evening. Topics of greatest interest included ways to be more physically active as a family, easy-to-fix meals, strategies to prepare food with their child/ren, and affordable home meals [34].

From these interview results we developed a family night protocol and procedures manual adapted from other school-based studies [35, 36]. Cooking stations and physical activities were designed using several constructs from SCT and ELT [22, 23]. We held 2 pilot events at a non-participating school, with half of the events tested at the first event and the remainder at the second. Surveyed parents and children gave the event high marks (e.g., $\geq 4.0$ on 5 point scales, data not shown), and process evaluation through observation and 
Program: Fuel for Fun: Cooking with Kids Plus Parent and Play

Situation: Children need direct experiences with healthful foods \& physical activity, \& reinforcing home \& (after-) school environments

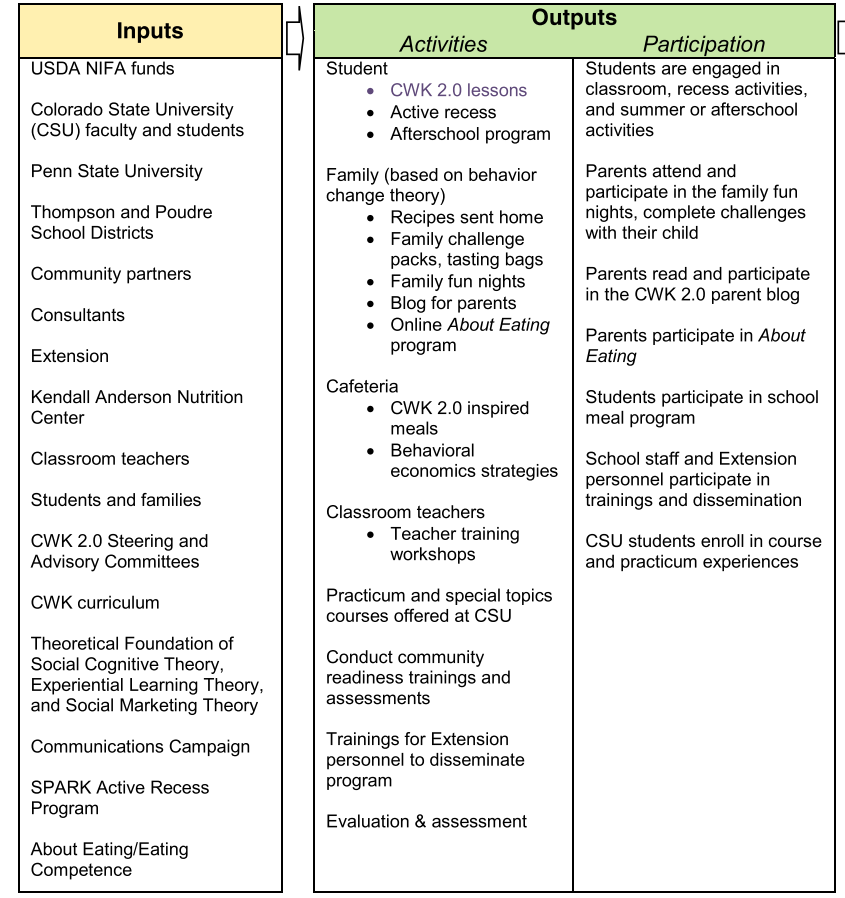

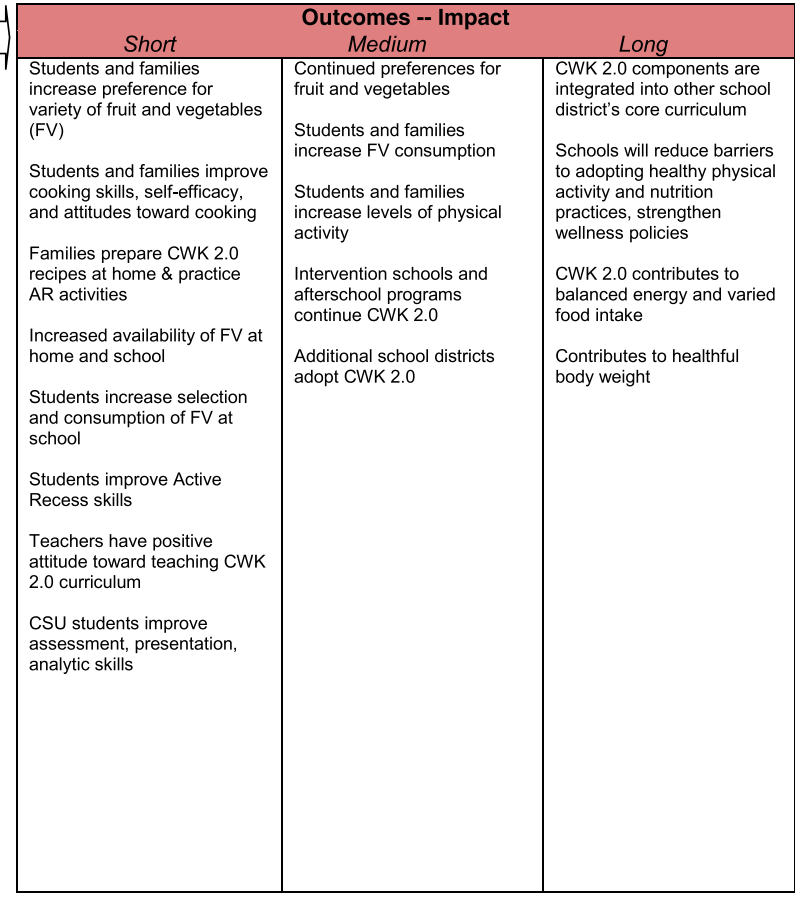

\section{Assumptions}

1) Schools/after-schools and parents are willing to fully participate in CWK 2.0

components; 2) CSU students are eager to enhance their education and gain hands on research experience; 3 ) Extension will adopt and disseminate the program

\section{External Factors}

School district budgets are constrained. Teachers face pressure to ensure students perform well on standardized tests. A school district's or Extension program's educational priorities may change.

Fig. 5 Fuel for Fun: Cooking with kids plus parents and play logic model

debriefing after each event identified minor revisions (e.g., refining necessary booth resources and improving event flow). Action packs and parent blogs were designed with educator and social media expert input respectively, to reinforce the content of the classroom cooking and tasting lessons and SPARK recess games. Final content of each intervention component is described below.

\section{Classroom}

The FFF classroom component - Cooking with Kids Colorado - includes 5 2-h CWK cooking and 5 1-h tasting lessons taught over the course of the academic year (i.e., 1 lesson approximately every 3 weeks). Lessons emphasize experiential learning of practical cooking skills using fresh and affordable foods from diverse cultural traditions [27]. Participating teachers are invited to an orientation session prior to the commencement of the program. Cooking and tasting lessons are taught in the classroom or cafeteria by graduate nutrition students trained as Food Educators, with assistance from the classroom teacher and another (graduate or undergraduate) nutrition student.

\section{Cafeteria}

FFF Cafeteria Connections includes lunch service of fruit and vegetable items that children prepare and taste in the classroom lessons, placing these fruits and vegetables prominently on the lunch line, highlighting them with colorful Fuel for Fun branded signage, and use of verbal prompts to encourage students to select fruits and vegetables. Cafeteria staff are trained to deliver these intervention components during the weeks that cooking and tasting lessons are delivered in the $4^{\text {th }}$-grade classrooms, and they also wear FFF chef coats or aprons on school days when implementing these strategies to further promote the program.

\section{SPARK active recess}

Since all $4^{\text {th }}$-grade students in the eight schools participate in lunch recess for approximately $20 \mathrm{~min}$ each day, this recess period offers an ideal opportunity for children to increase their physical activity without taking away from the school curriculum. Sports, Physical Activity and Recreation for Kids (SPARK) is an evidence-based program shown to increase children's participation, confidence and skills in physical activity [26]. Trained Active 
Recess Leaders (university Health and Exercise Science students) lead SPARK Active Recess activities a minimum of 4-days a week on the school playground during lunch recess. The noncompetitive games include "sharks and minnows", "2 square" and "cat and mice." Schools are provided with all activity equipment that supports the Active Recess program, such as balls, hoops, cones, jump ropes, bean bags, and flying discs.

\section{Fuel for Fun Family}

FFF Family is offered in those schools receiving the family component and includes three elements: Family Nights, Action Packs, and a parent blog. The FFF Family Night is scheduled two times each intervention year, fall and spring, and takes place at the participating school. Invitation flyers are sent home with students at least two weeks in advance, and two additional email reminders are sent to parents before the event. Activities include cooking and tasting stations that mimic what students experience in the classroom, food and nutrition crafts, and SPARK games children have learned at recess. After families rotate through each station, they are invited to enjoy a light meal provided and served by the school cafeteria staff. Meals are designed using project recipes. To motivate attendance and promote project goals, each Family Night closes with a drawing for free cooking and activity prizes.

The second family element, FFF Action Packs, is an activity packet sent home with students after each tasting and cooking lesson. The activities correspond with each classroom lesson and SPARK Active Recess games and include nutrition and physical activity sections. Participation of parents and other family members is strongly encouraged through written prompts and documented by parent signature. Students are reminded to return completed Action Packs by the following lesson. Lastly, the FFF Parent Blog is designed to engage parents and keep them up-to-date on the FFF activities going on in their child's school. The blog, which is slightly tailored for each school to align with the dates of FFF activities, also provides parents with tips about cooking with children, encouraging children to try new foods, and physical activities for the family. Content is posted to the private Facebook page weekly during the intervention, with reminders sent to parents to view the posts.

\section{About Eating}

About Eating is a web-based, self-directed, interactive program that addresses core constructs of the Satter model of eating competence [37], and focuses on food enjoyment and acceptance, attention to internal regulation, food resource management skills, and physical activity. It is learner-centered in that each of the 6 lessons can be viewed in any order, as often as desired, and with individually tailored depth and scope of participation. Iterative development and evaluation activities reveal that About Eating has face, process and content validity [21]. FFF parents in schools assigned to receive About Eating are invited to begin this program after they have completed their first survey (https://www.needscenter.org/resources1/about-eating/).

\section{Assessment and outcome measures}

A description of each measure is provided below. Refer to Table 1 for a list of measures and timing of data collection at the individual (child, parent), classroom, cafeteria, and playground levels.

\section{Child measures}

FFF student survey The FFF student survey in each participating classroom at 3 time points: Baseline (at the beginning of the school year prior to intervention, e.g., September), Follow-Up 1 (after intervention is completed in late spring of the same school year, e.g., May), and Follow-Up 2 (fall of the following school year, e.g., October). Standardized administration includes instructions and items read aloud as students complete the survey using pencil and paper. This survey set includes 35 cooking and fruit/vegetable items previously confirmed for validity and reliability [38]. Seven of these items address students' fruit preferences, 11 items for vegetable preference, 6 items for cooking attitudes, 8 items for cooking self-efficacy, and 3 items assess prior cooking experience.

Student report of physical activity is assessed through a 6-item adaptation of Godin \& Shephard's brief LeisureTime Exercise Questionnaire [39, 40], measuring days per week and daily minutes of mild, moderate and strenuous activity. Sedentary behavior is measured through students' estimate of daily hours of TV watching, video game playing and use of the Internet for non-homework activities [41]. PA stage of change is determined using Schumann and colleagues' measure [42, 43].

Height and weight Aligned with the FFF survey administration period, all participating children's height and weight are measured by trained research staff. Height is measured to the nearest tenth of a centimeter using a portable stadiometer (SECA, Model 213), and weight measured to the nearest tenth of a kilogram using a standard scale (Health o meter, Model 394KLX). Children are instructed to remove their shoes and any heavy clothing (e.g., jackets, sweatshirts tied around their waists, etc.) prior to measurement. Height is measured with children's arms by their sides and looking straight ahead. The 
$\frac{\text { Table } 1 \text { Evaluation measures for children and parents participating in the Fuel for Fun study }{ }^{\mathrm{a}}}{\text { Target measurement }}$

Description

Demographics Individual Level

Child age, birthdate, gender, race and ethnicity parent gender, age, race and ethnicity, nutrition and food assistance program participation, level of schooling, serious disease diagnosis

Height/weight

Child measured; parent self-report

Dietary intake assessment $(24-h \text { recalls })^{b}$

Physical activity

Student-telephone

Parent-online

7-day accelerometry (75 hz; GENEActiv)

Minutes/week of moderate-to-vigorous physica activity (MVPA); adaptation of Godin/Shephard questionnaire [62]

Screen time

Stage of change for regular physical activity $[42,43]$

International Physical Activity Questionnaire (IPAQ) [63]

Cooking experience

Cooking with Kids Student Survey [38]

Fruit and vegetable preferences

Cooking self-efficacy
Cooking with Kids Student Survey

Cooking with Kids Student Survey
Child information obtained from class rosters provided by schools; parent information is selfreported as part of an online parent survey

Child data collected by research team using standard protocol; parent self-reported as part of online survey

The Pennsylvania State University Diet Assessment Center protocol

7 days of free living, wrist-mounted accelerometry data from children and their parents; customized Matlab program will sum child and parent accelerations over 1 and $60 \mathrm{~s}$, respectively, and apply published GENEActiv cutpoints to determine amount of time in MVPA weekday, weekend day, and specific time periods (before school, school-day, after-school, and evening)

Students asked days/week and minutes/day of vigorous, moderate, and mild activity during free time; responses for vigorous and moderate PA summed for total MVPA.

Numbers of hours spent/day watching TV, playing video games or using a computer (not for homework). Responses 0-11 h

Students asked "Do you do regular physica activity as described?" Each of 5 responses correspond to one of the stages of Transtheoretical Model

Responses converted to met-min/week and identified as low, moderate, and high activity categories

Do you cook with family? Do you cook with friends? Do you cook? yes or no response options

Preference for 7 fruits and 11 vegetables; 18 items, 5 response options, scored from 1 to 5 , possible score 18-90. Cronbach's a 0.82

Self-efficacy for skills related to cooking; 8 items, 5 response options, scored from 1 to 5 , possible score $8-40$. Cronbach's a 0.70 
Table 1 Evaluation measures for children and parents participating in the Fuel for Fun study (Continued)

Cooking attitudes

Cooking with Kids Student Survey

Eating Competence:

Satter Eating Competence Inventory

(ecSI 2.0) [44]

Food resource management

skills

Culinographics (cooking

practices demographics)

Modeling eating behavior

Self-efficacy/Outcome

expectancies (SE/OE)

In-home fruit and vegetable availability

Parenting Style

Parent Perceived Stress

Plate waste assessment

Physical activity assessment/ observation ${ }^{\mathrm{e}}$
Expanded Food and Nutrition Education Program (EFNEP) adults core behavio checklist questions [64]

Questions from Krall and Lohse [44]

Modeling Scale. Sample items: How often

How often do you eat vegetables at dinner with your child? [65]

Perceived ability to offer fruits and vegetables that their child will eat. Sample item: I can prepare vegetables that my child will like. [66]

Fruit and vegetable availability inventory. [46]

Caregiver's Feeding Style Questionnaire [67]

Single item from the Community Health Database [68]

Group Level

Digital photography [51]

SOPLAY observation [26] do you eat dinner with your child?
Attitude toward cooking and making food: 6

Attitude 5 response 0

possible score 6-30.Cronbach's a 0.76

Parents: 16 items, 5 response options scored

from 3 to 0 . Possible score $0-48$; scores 32

indicate eating competence. Cronbach's a 0.89

Students: FU1 3 Eating attitudes and behavior

items; possible score 0-9

FU2 16 items, 5 response options scored from 3

to 0 Possible score $0-48$; scores 32 indicate

eating competence. Cronbach's a 0.89

13 items, 5 response options. Mean value for

each item

7 items, multiple choice

response options. Possible scores 0-33.

Cronbach's a 0.77

12-items modified from tested measure each

with 5 response options. Possible scores 12-60. Cronbach's a 0.93

20 items (fresh, frozen, canned fruits, vegetables and $100 \%$ juices) listed. Availability was affirmed or denied. Possible scores 0-20

19 items, 5 response options. Scores converted to 4 caregiver feeding styles.

Visual analog scale from 0 (no stress) to 10

(extreme stress).

Pre-consumption reference trays and post consumption trays photographed; plate waste of each food item estimated to nearest $10 \%$

Validated tool for direct observation of physical activity associated and environmental

characteristics in free play settings. MVPA and

estimates of energy expenditure are calculated

from activity counts

${ }^{\mathrm{a}}$ Measures collected at Baseline, month 7 and month 12

bietary intake assessment completed with a subsample of up to 100 parent/child dyads

cAccelerometry measured on a subsample of children and parents from 3 of the 8 participating schools

dPlate waste estimated from lunches of all assenting 4th-grade students participating the National School Lunch Program

eSystem of Observing Play and Leisure Activity in Youth; conducted at all 8 participating schools during lunch time recess 
research team returns within one week to collect measures from students absent on the day of testing.

\section{Parent measures}

FFF parent survey Consented parents complete an online survey at the same 3 time points as their child. For the first and second follow-up surveys, email reminders are sent within 2 to 4 weeks after the initial "evite." The survey includes demographics and valid measures of eating competence, [44], sense of coherence [45], modeling eating behaviors, self-efficacy, and fruit and vegetable availability [46]. Upon survey submission, parents are sent an animated, musical e-card with an e-gift card pin.

\section{Physical activity assessment of parent-child pairs using accelerometry}

Fourth-grade children and their parents in 3 randomly selected FFF schools are recruited to participate in the accelerometry (ACC) portion of the study. Using the wrist-mounted GENEActiv ACC recording at a sampling frequency of $75 \mathrm{~Hz}$ (ActivInsights Ltd., Cambridgeshire, UK), seven days of free-living data are collected. The device is attached to each child's non-dominant wrist using a semi-removable, hospital-style plastic band. Children are instructed to leave the device on for the entire seven-day period, even while sleeping and bathing. Parents and teachers are provided written instructions for children's ACC use, notably, that children should not tamper with the device nor remove it during the assessment period. Children are encouraged to engage in their typical daily activities while wearing the ACC, and teachers provide the research team a daily class schedule for the week of ACC wear, including school start and end times, lunch and recess times, and PE periods. Extra wristbands are provided in case a child has to remove the ACC for any reason. Children receive a $\$ 10$ gift-card to a local "superstore" upon receipt of the ACC as compensation for participation. Accelerometers, written instructions, and gift-cards for any consented children absent at drop-off are left with the teacher, with instructions to attach the device upon the child's return to school. At the end of the seven-day period, members of the research team return to collect the ACCs and the children receive a second gift card, this one valued at $\$ 15$. Children must return their device and the device worn by their parent, if applicable, to receive the $\$ 15$ gift card.

Parents who consent for themselves and their child to the ACC portion of the study receive a packet with one ACC, semi-removable plastic ACC wristbands, and ACC instructions, which detail how to attach the device and frequently asked questions regarding the ACC and ACC-wear. This packet is sent home with the child on the ACC drop-off day, and children are asked to give the ACC to their parents as soon as they get home. Similar to the child protocol, parents are asked to wear the device for seven days and to not tamper with or remove the ACC. Parents are instructed to return the device to school via their child on ACC pick-up day. There is no separate compensation for parents to participate in the ACC portion of the study. These parents are also asked to complete a demographic information form surveying their sex, height, weight, and age.

\section{Diet assessment of parent-child pairs using 24-h dietary recalls}

At the baseline of project years 2 through 4, following completion of the parent survey, we are recruiting parents to participate with their child in a diet assessment (DA) protocol for each of the three data collection time points described above. Child DA consists of three 24-h dietary recalls collected by telephone over a $2-4$ week period by trained interviewers at the Pennsylvania State University Diet Assessment Center. Intake data are collected using Nutrition Data System for Research software versions 2013 and 2014, developed by the Nutrition Coordinating Center (NCC), University of Minnesota, Minneapolis, MN (http://www.ncc.umn.edu). This time-related database updates analytic data while maintaining nutrient profiles true to the version used for data collection [47]. These child-oriented interviews are conducted with the parent present in most cases, and typically last 15 to $20 \mathrm{~min}$. Calls are unannounced with a goal of obtaining two weekday and one weekend dietary recalls.

Upon completion of three telephone dietary recalls for the child's intake, we contact parents via email to complete online dietary recalls for themselves using the National Cancer Institute's Automated Self-administered 24-h recall (ASA24) software [48-50]. Email requests are also unannounced with a goal of obtaining two weekday and one weekend dietary recalls. If parents are unresponsive to email requests, telephone contact is made to ensure they were receiving the requests and to encourage participation. Parent dietary intake data are collected and analyzed using the ASA24 system, version 2011 [50]. We send parents e-gift cards for each DA completed; values increase incrementally for each of the 3 DA. E-gift card pins are embedded in an online animated, musical card.

\section{School-level outcome measures}

Plate waste assessment To assess the impact of FFF on student fruit and vegetable intake during school lunch, the selection and consumption of all foods obtained from the school cafeteria during the $4^{\text {th }}$-graders' lunch 
period is measured using a tested digital photography plate waste method [51-54]. Plate waste is assessed on four occasions throughout the school year; once at baseline and three times after implementation of the intervention. Only fourth-graders purchasing a National School Lunch Program reimbursable meal (approximately $50 \%$ of all $4^{\text {th }}$-graders) are invited to participate. Photographs taken of five samples of each menu item serve as the "before" reference photographs. Two independent analysts estimate the difference of each menu item from the "after" photo of each student's lunch tray compared to the "before" photos to the nearest $10 \%$.

System for Observing Play and Leisure Activity in Youth (SOPLAY) We use the SOPLAY procedure [55] to document the number of students and their physical activity levels on the school playground during lunch recess. Each school playground is divided into distinct scan areas (e.g., swing sets, basketball court, open field). During a scan, each child's activity is mechanically coded as sedentary (e.g., standing, sitting), walking, or very active (e.g., running, jumping). Separate scans are made for boys and girls. Predominant activity type (e.g., climbing, soccer) and characteristics of each area are noted, including accessibility, usability, and presence of supervision, organized activities and equipment. Time of day, day of week, temperature, weather conditions, and other variables that can impact the length of recess or children's physical activity levels are also recorded. During each of three measurement periods for each cohort, four lunch time recesses are observed at each school over 10 school days (2 weeks), resulting in a total of 32 observation days per measurement period. Reliability data will be collected during eight of these 32 observation days ( $25 \%$ ) by pairs of observers who will make simultaneous and independent observations.

Teacher measures After the completion of the intervention, teachers are emailed an invitation to complete an online survey. Survey items were adapted from a previously validated instrument for classroom teachers [56], with content validity confirmed by expert panel and face validity and comprehension confirmed through cognitive interviews with 4 elementary school teachers from the same district, but not participating in Fuel for Fun [57]. Survey items include beliefs about the school nutrition environment, including who is responsible for that environment, teachers' abilities to influence the nutrition environment, and the importance of role modeling healthful nutrition behaviors. Other survey content includes eating competence [44], education level and years of teaching experience, cooking attitudes and behaviors and college nutrition course participation. Upon survey completion they are given a thank you note and \$25 gift card.

\section{Process measures}

- Food Educators complete a debriefing form at the conclusion of each classroom cooking and tasting lesson to record numbers of students present, student recollections from the previous lesson, student and classroom teacher engagement, and any issues encountered or changes to the lesson.

- Research team members observe each Food Educator four times during the school year to assess fidelity to the lesson plan, any issues observed, and Food Educator, student and teacher engagement [58]. Clarification of expectations is provided to the Food Educator as needed to confirm consistency of lesson delivery.

- SPARK leaders complete a debriefing form after each active recess to document number of students, length of recess, weather conditions, SPARK activities implemented, and any issues encountered. Each SPARK leader is observed five times throughout the school year by a member of the research team to document fidelity to the activity plan.

- School cafeteria staff are observed by members of the evaluation team during lunch four times throughout the school year to document implementation of the Cafeteria Connection component.

- Sign-in sheets for students and their families document participation in Family Night events. The Family Night event coordinator records recruitment, fidelity to the planned activities, and overall impressions of the event. Parents and students complete a short survey addressing their attitudes toward the Family Night activities.

- Parent engagement in the Action Pack activities is assessed with a parent signature on each returned action pack and researcher review to determine number of action pack completions.

- Research staff monitor timing, content and participation in the parent blog posts.

- About Eating participation is monitored by website analytics and survey administered immediately after each lesson.

- District and school-level data are requested annually from wellness coordinators that describe the nutrition and activity environments at each school. These include length of lunch periods, frequency and length of physical education classes, whether recess occurs before or after lunch, and description of any nutrition education topics or programs taught during or after school.

\section{Analysis plan}

Interview data and other qualitative process data are analyzed using Atlas.ti (Cleverbridge, Inc; Chicago, IL) and 
NVivo (QSR International; Burlington, MA) to conduct content and thematic analyses following transcription of audio records. SOPLAY data are ranked and categorized as suggested by McKenzie and colleagues [26]. Scores are calculated as directed for each adult and child survey. Following determination of or transformation to a normal distribution and assessment of survey internal consistency, data are analyzed using a repeated measures general linear model that controls for significant group (co-factor) or characteristic (covariate) differences. Cluster analyses will also be conducted to assist with data interpretation.

Power calculations for primary outcomes are based on previous studies with $4^{\text {th }}$ grade Colorado students and parents. Minimum sample sizes to detect a clinically relevant change at a power of 0.80 with an alpha of 0.05 are shown in Table 2 below. According to experts at The Pennsylvania State University Diet Assessment Center, datasets are useful when at least 75 individuals have completed at least 2 of the 3 diet recalls for each measurement period.

\section{Accelerometry}

Child ACC data are downloaded with the GENEActiv PC Software (ActivInsights Ltd., Cambridgeshire, UK). Using a custom $\mathrm{R}$ script ( $\mathrm{R}$ Foundation for Statistical Computing, Vienna, Austria), data are low-pass filtered at $15-\mathrm{Hz}$, vector summed, and corrected for gravity by subtracting one. Values are then averaged over $75 \mathrm{~Hz}$ to obtain one value per second of wear-time. Next, a custom Matlab program (Mathworks, Inc., Natick, MA) and previously determined cutpoints [59] are used to process the acceleration data and output minutes spent in activities of sedentary, light, moderate, and vigorous intensities for each day that the device was worn and for

Table 2 Sample size requirements for power levels of 0.8 and 0.9 based on prior studies of Cooking with Kids

\begin{tabular}{lllll}
\hline Measure & $\begin{array}{l}\text { Clinically } \\
\text { relevant } \\
\text { change }\end{array}$ & \multicolumn{2}{l}{ Minimum sample size/group } \\
\cline { 4 - 5 } & 2 & 50 & $1-\beta=0.9$ \\
\hline Student attitude & 2 & 108 & 66 \\
Student self-efficacy & 3 & 127 & 144 \\
Student FV preference & 3 & 64 & 170 \\
Parent modeling & 2 & 110 & 146 \\
Parent self-efficacy & 4 & 15 & 20 \\
Parent FV availability & 2 & 105 & 140 \\
Parent eating competence & 3 &
\end{tabular}

${ }^{\mathrm{a}}$ Based on repeated pre/post measures using standard deviations from previous research. For example, a total of 100 participants are needed in this two-treatment parallel-design study. The probability is $80 \%$ that the study will detect a treatment difference in student attitude at a two-sided 0.05 significance level, if the true difference between treatments is 2 units. This is based on the assumption that the standard deviation of the response variable is 3.5 website: http://hedwig.mgh.harvard.edu/sample_size/js/js_parallel_quant.html specific intervals of the day (i.e., full-day, before school, school-day, recess, PE, after-school, evening). This program also identifies periods of time when the device was not worn (any period of $>60 \mathrm{~min}$ during which ACC output was $<0.06$ gs or any period that was identified as having $\geq 98 \%$ of the interval indicated as sedentary). To be included in analyses, children are required to have at least four valid days of ACC-wear, with a valid day including at least $600 \mathrm{~min}$ of ACC wear-time. Any days with less than 600 min of wear and any children with fewer than four valid days are removed from the dataset.

Like the child data, parent ACC data are downloaded with the GENEActiv PC Software (ActivInsights Ltd., Cambridgeshire, UK), and a custom Matlab script ( $R$ Foundation for Statistical Computing, Vienna, Austria) is used to vector sum and correct the data for gravity by subtracting one. Values are then summed over 60-s to obtain one value per minute of wear-time. Next, a custom Matlab (Mathworks, Inc., Natick, MA) program and previously published cutpoints [60] are used to process the acceleration data and output minutes spent in activities of sedentary, light, moderate, and vigorous intensities for each day that the device was worn and for specific intervals of the day (i.e., full-day, before school, school-day, after-school, evening, late-evening). This program also identifies periods of time when the device was not worn (any period of $>60$ min during which ACC output was $<100$ gs or any period that was identified as having $\geq 98 \%$ of the interval indicated as sedentary). To be included in analyses, parents are required to have at least four valid days of ACC-wear, with a valid day including at least $600 \mathrm{~min}$ of ACC wear-time. Any days with less than 600 min of wear and any parents with fewer than four valid days are removed from the dataset.

\section{Quality control and data management}

Research staff and university students are trained in intervention implementation and/or data collection and entry as appropriate. Student survey and height/weight data are dual entered, then compared using SPSS by a third research team member to confirm accuracy. Parent data are downloaded from Qualtrics [61], reviewed to assure eligibility and compared with incentive payment records. All datasets are maintained on a password protected, secure University server.

\section{Discussion}

The Fuel for Fun: Cooking with Kids Plus Parents and Play logic model depicts the breadth of resources, inputs, and activities designed to achieve immediate and longer-term outcomes to effectively prevent childhood obesity within study participants (Fig. 5). The study design (Fig. 1) includes 4 annual cohorts (2 student control and 2 intervention) in 8 schools from 2 school districts, 
with 2 parent groups in the control cohorts and 4 parent groups in the intervention cohorts. All students receive the school-based classroom, cafeteria, and active recess components. Parent group involvement includes a range of activities from control to both FFF family involvement and About Eating, an online food resource management program.

Unique strengths of this study include significant opportunities for parent involvement, and examination of parent engagement and parent response to 1 of 4 treatment options. Other significant contributions include the use of accelerometry, dietary, and survey data; with parent-child dyads for accelerometry and dietary assessments. Unlike many childhood obesity prevention interventions, we will follow students and parents into the subsequent school year, collecting a full year of diet and physical activity-related information. Additionally, the intervention and control groups are randomly assigned and cohort-based. Our dissemination plan includes adapting and testing FFF in afterschool and other out-of-school based venues and for low-income audiences.

This project builds on our prior work that shows impact of the $C W K$ intervention on 4th graders, especially boys without previous cooking experience for both Hispanic [7] and mainly Caucasian samples [6]. In addition, eating competence, shown to be associated with parent feeding and food-related behaviors in prior Hispanic sample [46], is included in the assessment of this mostly Caucasian, socioeconomically diverse sample of parents. Cafeteria Connections builds on previous school cafeteria behavioral economics research by Wansink and Just [24, 33] and ties the strategies to the FFF classroom lessons. Moreover, valid instruments and procedures are used to collect all study data.

In addition to examining the impact of an effective cooking and tasting curriculum on student foodrelated behaviors, we will examine parent engagement, interplay between parent and child involvement, relationships between physical activity, diet, and eating behaviors, and sustainability and dissemination potential of this type of curriculum. We will also explore and characterize the psychometric properties of previously tested instruments about physical activity, food and eating behaviors.

Limitations include that the target audience lacks racial and ethnic diversity, thereby restricting the generalizability of results. Because of the intervention design, we will have only indirect involvement of parents, relying on them to accept our invitations to join their child in intervention and measurement activities. If teachers continue teaching 4th grade in participating schools, they will participate in both control and intervention phases of the project; their prior experience may influence subsequent years. We will not be able to follow students beyond the beginning of the 5th grade year, therefore long-term impact is unknown.

\section{Abbreviations \\ ACC, accelerometry; ASA24, Automated Self-administered 24-h recall; CWK Cooking with Kids; DA, diet assessment; ELT, Experiential Learning Theory; FFF, Fuel for Fun; PA, physical activity; SCT, Social Cognitive Theory; SEM, Social Ecological Model; SOPLAY, System for Observing Play and Leisure Activity in Youth; SPARK, Sports, Physical Activity and Recreation for Kids}

\section{Acknowledgements}

We extend our sincere thanks to members of the Fuel for Fun steering committee not co-authoring this manuscript: Susan Baker and Laura Bellows at Colorado State University, Laurie Zenner with the University of Colorado Healthy Kids Club, and Lynn Walters and Jane Stacy with Cooking with Kids, Inc. We also thank Diane Mitchell and Kristen A. Cox with the Pennsylvania State University Diet Assessment Center, our project advisory committee, graduate and undergraduate research assistants, and the students, parents, teachers, cafeteria staff and administrators of participating Fuel for Fun schools. We also thank Jessica Clifford, Melissa Pflugh Prescott, Alixanna Burg, and Kathryn Faulring for their manuscript preparation support.

\section{Funding}

This material is based on work supported by the National Institute of Food and Agriculture, U.S. Department of Agriculture, under award number 201268001-19603.

\section{Availability of data and material}

No additional data is referenced beyond that described in the manuscript.

\section{Authors' contributions}

LCS is the PI and along with $B L$, the co-PI, developed the initial draft of this manuscript. LCS, BL, MB, RB, KK, and CN contributed to the conception and design of the study. All authors, including ES and ER, contributed to manuscript development. All authors have read and approve the final manuscript.

\section{Authors' information}

No additional information is necessary.

\section{Competing interests}

The authors declare that they have no competing interests.

\section{Consent for publication}

No individual data or information is provided, thus negating need for individual's consent for publication.

\section{Ethics approval and consent to participate}

This project was given expedited approval by Colorado State University's Research Integrity and Compliance Review Office; protocol number 12$3278 \mathrm{H}$. Informed consent (assent for children) was obtained from all study participants.

\section{Author details}

'Department of Food Science and Human Nutrition, Colorado State University, 234 Gifford Building, Fort Collins, Colorado 80523-1571, USA.

${ }^{2}$ Wegmans School of Nutrition and Health, Rochester Institute of Technology, Rochester, New York 14623, USA. ${ }^{3}$ Department of Food Science and Human Nutrition, Colorado State University, 1571 Campus Delivery, Fort Collins, Colorado 80523-1571, USA. ${ }^{4}$ Nike, Inc., Beaverton, Oregon 97005, USA. ${ }^{5}$ Aims Community College, 5401 W 20th, Greeley, Colorado 80634, USA.

${ }^{6}$ Department of Public Health Studies, University of Hawaii at Manoa, 1960 East-west Road, Honolulu, Hawaii 96822, USA. 'Department of Biology, Colorado State University, 1878 Campus Delivery, Fort Collins, Colorado 80523-1878, USA. ${ }^{8}$ Department of Marketing, Colorado State University, 1278 Campus Delivery, Fort Collins, Colorado 80523-1278, USA. 


\section{Received: 11 March 2016 Accepted: 14 May 2016}

\section{Published online: 26 May 2016}

\section{References}

1. Wang Y, Wu Y, Wilson RF, Bleich S, Cheskin L, Weston C, Showell N, Fawole O, Lau B, Segal J. Childhood obesity prevention programs: comparative effectiveness review and meta-analysis. In: Comparative Effectiveness Reviews. Rockville: Agency for Healthcare Research and Quality; 2013.

2. Nelson SA, Corbin MA, Nickols-Richardson SM. A call for culinary skills education in childhood obesity-prevention interventions: current status and peer influences. J Acad Nutr Diet. 2013;113(8):1031-6.

3. Condrasky MD, Hegler M. How culinary nutrition can save the health of a nation. J Ext. 2010;48(2):1-6.

4. Groffik D, Sigmund E, Frömel K, Chmelík F, Lokvencová PN. The contribution of school breaks to the all-day physical activity of 9-and 10-year-old overweight and non-overweight children. Int J Public Health. 2012;57(4):711-8.

5. Hersch D, Perdue L, Ambroz T, Boucher JL. The impact of cooking classes on food-related preferences, attitudes, and behaviors of school-aged children: a systematic review of the evidence, 2003-2014. Prev Chronic Dis. 2014;11:E193.

6. Cunningham-Sabo L, Lohse B. Cooking with kids positively affects fourth graders' vegetable preferences and attitudes and self-efficacy for food and cooking. Child Obes. 2013;9(6):549-56.

7. Cunningham-Sabo L, Lohse B. Impact of a school-based cooking curriculum for fourth-grade students on attitudes and behaviors is influenced by gender and prior cooking experience. J Nutr Educ Behav. 2014;46(2):110-20.

8. Cooking with Kids. [http://cookingwithkids.org/]. Accessed 15 Jul 2015.

9. Lubans DR, Plotnikoff RC, Morgan PJ, Dewar D, Costigan S, Collins CE. Explaining dietary intake in adolescent girls from disadvantaged secondary schools. A test of Social Cognitive Theory. Appetite. 2012;58(2):517-24.

10. Blanchette L, Brug J. Determinants of fruit and vegetable consumption among 6-12-year-old children and effective interventions to increase consumption. J Hum Nutr Diet. 2005;18(6):431-43.

11. De Bourdeaudhuij I, te Velde S, Brug J, Due P, Wind M, Sandvik C, Maes L, Wolf A, Rodrigo CP, Yngve A et al. Personal, social and environmental predictors of daily fruit and vegetable intake in 11-year-old children in nine European countries. Eur J Clin Nutr. 2007;62(7):834-41.

12. Gibson CA, Smith BK, DuBose KD, Greene JL, Bailey BW, Williams SL, Ryan JJ, Schmelzle KH, Washburn RA, Sullivan DK et al. Physical activity across the curriculum: year one process evaluation results. Int J Behav Nutr Phys Activ. 2008:5(1):1-11.

13. Dale D, Corbin CB, Dale KS. Restricting opportunities to be active during school time: do children compensate by increasing physical activity levels after school? Res Q Exerc Sport. 2000;71(3):240-8.

14. Sallis JF, McKenzie TL, Alcaraz JE, Kolody B, Faucette N, Hovell MF. The effects of a 2-year physical education program (SPARK) on physical activity and fitness in elementary school students. Sports, Play and Active Recreation for Kids. Am J Public Health. 1997;87(8):1328-34.

15. Huang TT, Drewnowski A, Kumanyika SK, Glass TA. A systems-oriented multilevel framework for addressing obesity in the 21st century. Prev Chronic Dis. 2009;6(3):A82.

16. Hoelscher DM, Kirk S, Ritchie L, Cunningham-Sabo L. Position of the Academy of Nutrition and Dietetics: interventions for the prevention and treatment of pediatric overweight and obesity. J Acad Nutr Diet. 2013;113(10):1375-94.

17. Cluss PA, Fee L, Culyba RJ, Bhat KB, Owen K. Effect of food service nutrition improvements on elementary school cafeteria lunch purchase patterns. J Sch Health. 2014;84(6):355-62.

18. Golan M, Kaufman V, Shahar DR. Childhood obesity treatment: targeting parents exclusively v. parents and children. Brit J Nutr. 2006;95(5):1008-15.

19. McLeroy KR, Bibeau D, Steckler A, Glanz K. An ecological perspective on health promotion programs. Health Educ Q. 1988;15(4):351-77.

20. Dietary Guidelines for Americans 2015-2020, 8th edition. [http://health.gov/ dietaryguidelines/2015/guidelines/]. Accessed 1 Mar 2016.

21. Lohse B, Belue R, Smith S, Wamboldt P, Cunningham-Sabo L. About eating: an online program with evidence of increased food resource management skills for low-income women. J Nutr Educ Behav. 2015;47(3):265-72. e261.

22. McAlister AL, Perry CL, Parcel GS. How individuals, environments, and health behaviors interact. In: Health behavior and health education: theory research and practice. 4th ed. San Francisco: Jossey-Bass; 2008. p. 169-88.

23. Kolb D. Experiential learning: experiences as the source of learning and development. Englewood Cliffs: Prentice-Hall Inc; 1984.
24. Just DR, Wansink B. Smarter lunchrooms: using behavioral economics to improve meal selection. Choices. 2009;24(3):1-7.

25. Evans WD, Christoffel KK, Necheles JW, Becker AB. Social marketing as a childhood obesity prevention strategy. Obesity. 2010;18(S1):S23-6.

26. McKenzie T, Rosengard P, Short K, Strelow J. Sports, Play and Active Recreation for Kids active recreation manual ages 5-14. San Diego: State University Foundation; 2000.

27. Walters LM, Stacey JE. Focus on food: development of the cooking with kids experiential nutrition education curriculum. J Nutr Educ Behav. 2009; 41(5):371-3.

28. Wiggins G, McTighe J. The Understanding by design guide to advanced concepts in creating and reviewing units. Alexandria: ASCD; 2012.

29. Cunningham-Sabo L, Balgopal M, Walters L, Lohse B. Application of the understanding by design model in the adaptation of the Cooking with Kids curriculum to encourage classroom teacher acceptance and use. In: International Society of Behavioral Nutrition and Physical Activity Annual Meeting: 2014. San Diego: International Society of Behavioral Nutrition and Physical Activity; 2014. https://www.isbnpa.org/files/annual_meetings/2014/ 10/13/15/attachments/543cfbedef96.pdf.

30. Auld GW, Romaniello C, Heimendinger J, Hambidge C, Hambidge M. Outcomes from a school-based nutrition education program using resource teachers and cross-disciplinary models. J Nutr Educ. 1998;30(5):268-80.

31. Liquori T, Koch PD, Contento IR, Castle J. The Cookshop Program: outcome evaluation of a nutrition education program linking lunchroom food experiences with classroom cooking experiences. J Nutr Educ. 1998;30(5):302-13.

32. Prelip M, Slusser W, Thai CL, Kinsler J, Erausquin JT. Effects of a school-based nutrition program diffused throughout a large urban community on attitudes, beliefs, and behaviors related to fruit and vegetable consumption. J Sch Health. 2011;81(9):520-9.

33. Just DR, Wansink B, Mancino L, Guthrie J. Behavioral economic concepts to encourage healthy eating in school cafeterias. In: Economic Research Report no ERR-68. Washington, DC: US Department of Agriculture, Economic Research Service; 2008

34. Cheng F, Smith S, Cunningham-Sabo L, Lohse B. Telephone interviews with parents of 4 th graders support nutrition education using innovative strategies for low-income families and after-school programs FASEB J. 2014; 28(1 Supplement):625.5.

35. Davis SM, Clay T, Smyth M, Gittelsohn J, Arviso V, Flint-Wagner H, Rock BH, Brice RA, Metcalfe L, Stewart D et al. Pathways curriculum and family interventions to promote healthful eating and physical activity in American Indian schoolchildren. Prev Med. 2003;37(Supplement 1):S24-34.

36. Luepker RV, Perry CL, McKinlay SM, et al. Outcomes of a field trial to improve children's dietary patterns and physical activity: The child and adolescent trial for cardiovascular health (catch). J Am Med Assoc. 1996;275(10):768-76.

37. Satter E. Eating competence: nutrition education with the satter eating competence model. J Nutr Educ Behav. 2007;39(5):S189-94.

38. Lohse B, Cunningham-Sabo L, Walters LM, Stacey JE. Valid and reliable measures of cognitive behaviors toward fruits and vegetables for children aged 9 to 11 years. J Nutr Educ Behav. 2011;43(1):42-9.

39. Godin G, Shephard R. A simple method to assess exercise behavior in the community. Can J Appl Sport Sci. 1985;10(3):141-6.

40. Godin G, Jobin J, Bouillon J. Assessment of leisure time exercise behavior by self-report: a concurrent validity study. Can J Public Health. 1985;77(5):359-62.

41. Buckworth J, Nigg C. Physical activity, exercise, and sedentary behavior in college students. J Am Coll Health. 2004;53(1):28-34.

42. Schumann A, Nigg CR, Rossi JS, Jordan PJ, Norman GJ, Garber CE, Riebe D, Benisovich SV. Construct validity of the stages of change of exercise adoption for different intensities of physical activity in four samples of differing age groups. Am J Health Promot. 2002;16(5):280-7.

43. Haas S, Nigg CR. Construct validation of the stages of change with strenuous, moderate, and mild physical activity and sedentary behaviour among children. J Sci Med Sport. 2009;12:586-91.

44. Krall JS, Lohse B. Validation of a measure of the Satter eating competence model with low-income females. Int J Behav Nutr Phys Activ. 2011;8:26.

45. Wainwright NWJ, Surtees PG, Welch AA, Luben RN, Khaw K-T, Bingham SA. Healthy lifestyle choices: could sense of coherence aid health promotion? J Epidemiol Community Health. 2007;61(10):871-6.

46. Lohse B, Cunningham-Sabo L. Eating competence of Hispanic parents is associated with attitudes and behaviors that may mediate fruit and vegetable-related behaviors of 4th grade youth. J Nutr. 2012;142(10): 1903-9. 
47. Schakel SF. Maintaining a nutrient database in a changing marketplace: keeping pace with changing food products - a research perspective. J Food Composition Anal. 2001;14(3):315-22.

48. Schakel S, Sievert Y, Buzzard I. Sources of data for developing and maintaining a nutrient database. J Am Diet Assoc. 1988;88(10):1268-71.

49. Schakel SF, Buzzard IM, Gebhardt SE. Procedures for estimating nutrient values for food composition databases. J Food Composition Anal. 1997; 10(2):102-14.

50. Automated Self-Administered 24-h Recall (ASA24). https://asa24.nci.nih.gov.

51. Smith SL, Cunningham-Sabo L. Food choice, plate waste and nutrient intake of elementary- and middle-school students participating in the US National School Lunch Program. Public Health Nutrition. 2014;17(6):1255-63.

52. Martin CK, Newton Jr RL, Anton SD, Allen HR, Alfonso A, Han H, Stewart T, Sothern M, Williamson DA. Measurement of children's food intake with digital photography and the effects of second servings upon food intake. Eat Behav. 2007;8(2):148-56.

53. Swanson M. Digital photography as a tool to measure school cafeteria consumption. J Sch Health. 2008;78(8):432-7.

54. Williamson DA, Allen HR, Martin PD, Alfonso AJ, Gerald B, Hunt A. Comparison of digital photography to weighed and visual estimation of portion sizes. J Am Diet Assoc. 2003;103(9):1139-45.

55. McKenzie TL, Sallis JF, Broyles SL, Zive MM, Nader PR, Berry CC, Brennan JJ. Childhood movement skills: predictors of physical activity in Anglo American and Mexican American adolescents? Res Q Exerc Sport. 2002; 73(3):238-44.

56. Girard BL. Teacher attitudes, perceived influences, and self-reported classroom behaviors related to school nutrition environments. 2010.

57. Cunningham-Sabo L, Addington R, Lohse B, Balgopal M, Smith SL. Variability in Teachers' Nutrition Beliefs and Practices in the Elementary School Environment Warrant Attention in Impact Assessment. In: Poster presented at: International Society of Behavioral Nutrition and Physical Activity Annual Meeting: 2015. Edinburg: International Society of Behavioral Nutrition and Physical Activity; 2015.https://www.isbnpa.org/files/annual_meetings/2015/ 06/10/16/attachments/557869344fefe.pdf

58. Cunningham-Sabo L, Hanika-Ortiz A, Walters L, Stacey J, Lohse B. Process evaluation measures of experiential foods program for elementary schoolchildren. In: American Public Health Association Annual Conference: 2008. San Diego: American Public Health Association; 2008. https://apha. confex.com/apha/136am/webprogram/Paper178625.html.

59. Schaefer CA. Physical activity: improvement assessment tools and behavior in children. Fort Collins, Colorado, USA: Colorado State University; 2014.

60. Esliger DW, Rowlands AV, Hurst TL, Catt M, Murray P, Eston RG. Validation of the GENEA Accelerometer. Med Sci Sports Exerc. 2011;43(6):1085-93.

61. Qualtrics. Qualitrics software. Provo. 2015. https://www.qualtrics.com/.

62. Harmon BE, Nigg CR, Long C, Amato K, Anwar M, Kutchman E, Anthamatten P, Browning RC, Brink L, Hill JO. What matters when children play: influence of social cognitive theory and perceived environment on levels of physical activity among elementary-aged youth. Psychol Sport Exerc. 2014;15:272-9.

63. Craig $C L$, Marshall AL, Sjostrom M, et al. International physical activity questionnaire: 12-country reliability and validity. Med Sci Sports Exerc. 2003; 35:1381-95.

64. EFNEP Evaluation- Adult Program. [http://nifa.usda.gov/resource/efnepevaluation-adult-program]. Accessed 1 Mar 2016.

65. Cullen KW, Baranowski T, Rittenberry I, et al. Child-reported family and peer influences on fruit, juice and vegetable consuption: reliability and validity of measures. Health Educ Res. 2001;16:187-200.

66. Cullen KW, Baranowski T, Rittenberry I, et al. Socioenvironmental influences on children's fruit, juice and vegetable consumption as reported by parents: reliability and validity of measures. Public Health Nutr. 2000;3:345-56.

67. Hughes SO, Power TG, Risher JO, Mueller S, Nicklas TA. Revisiting a neglected construct: parenting styles in a child-feeding title. Appetite. 2005;44:83-92.

68. Parks EP, Kumanyika S, Moore $\mathrm{RH}$, et al. Influence of stress in parents on child obesity and related behaviors. Pediatrics. 2012;130:1096-104.

\section{Submit your next manuscript to BioMed Central and we will help you at every step:}

- We accept pre-submission inquiries

- Our selector tool helps you to find the most relevant journal

- We provide round the clock customer support

- Convenient online submission

- Thorough peer review

- Inclusion in PubMed and all major indexing services

- Maximum visibility for your research

Submit your manuscript at www.biomedcentral.com/submit
Biomed Central 